\title{
Índices ecoDopplercardiográficos de função ventricular esquerda em cães das raças Boxer e Schnauzer Miniatura
}

[EchoDopplercardiographic indexes of left ventricular function in Boxer and Miniature Schnauzer dogs]

\author{
E.F. Silva ${ }^{1}$, M.B. Melo ${ }^{1}$, R.A.L. $M u z z i^{2}$, R.B. Araújo ${ }^{1}$, R.C.S. Tôrres $^{1 *}$ \\ ${ }^{1}$ Escola Veterinária - UFMG \\ Caixa Postal 567 \\ 30123-970 - Belo Horizonte, MG \\ ${ }^{2}$ Departamento Medicina Veterinária - UFLA - Lavras, MG
}

\begin{abstract}
RESUMO
Avaliaram-se o índice de performance do miocárdio (IPM) e outros índices ecoDopplercardiográficos de função ventricular em cães de duas raças de diferentes biotipos. Foram utilizados 24 cães da raça Schnauzer Miniatura, com média de peso de $8,4 \pm 1,6 \mathrm{~kg}$, e 24 cães da raça Boxer, com média de peso de $25,1 \pm 2,6 \mathrm{~kg}$. O IPM na raça Schnauzer Miniatura foi 0,32 e na raça Boxer 0,48. Os valores do IPM, dos índices de fase de ejeção, do período de pré-ejeção, da relação período de pré-ejeção/período de ejeção e do tempo de desaceleração da onda $\mathrm{E}$ do fluxo mitral diferiram entre as duas raças. Observou-se correlação entre o peso corporal e esses índices, e o peso corporal foi considerado a principal característica racial responsável pelas diferenças observadas. $\mathrm{O}$ tempo de ejeção $(\mathrm{r}=-0,51)$, o período de pré-ejeção $(\mathrm{r}=-0,44)$ e o tempo de relaxamento isovolumétrico $(\mathrm{r}=-0,38)$ foram os únicos parâmetros a apresentar correlação com a freqüência cardíaca (FC). O uso da relação período de pré-ejeção/tempo de ejeção e do tempo de ejeção corrigido pela freqüência cardíaca diminui o efeito da $\mathrm{FC}$ sobre esses parâmetros.
\end{abstract}

Palavras-chave: cão, performance do miocárdio, função ventricular, ecoDopplercardiografia

\begin{abstract}
The index of myocardial performance (IMP) and others ecoDopplercardiographic indexes of ventricular function were evaluated in dogs of two different breeds and somatotypes. Twenty-four Miniature Schnauzer dogs averaging $8.4 \pm 1.6 \mathrm{~kg}$ and 24 Boxer dogs averaging $25.1 \pm 2.6 \mathrm{~kg}$ were used. IPM, ejection phase indexes, pre-ejection period, pre-ejection period/ejection time relation, and $E$ wave deceleration time of mitral inflow showed correlation to body weight with significant differences between the breeds. Body weight was considered the main breed characteristic responsible for the observed differences. Ejection time $(r=-0.51)$, pre-ejection time $(r=-0.44)$, and isovolumetric relaxation time $(r=-0.38)$ presented correlation with heart rate. The use of pre-ejection period/ejection time relation and ejection time correct by heart rate diminishes the influence of heart rate on the parameters.
\end{abstract}

Keywords: dog, myocardial performance, ventricular function, ecoDopplercardiography

\section{INTRODUÇÃO}

Na rotina clínica de pequenos animais, observase que várias alterações cardíacas determinam disfunção ventricular e, conseqüentemente,

Recebido em 25 de maio de 2007

Aceito em 31 de janeiro de 2008

*Autor para correspondência (corresponding author)

E-mail: rtorres@vet.ufmg.br

Apoio: FAPEMIG provocam um quadro de insuficiência cardíaca. Uma das principais contribuições da ecoDopplercardiografia é a possibilidade de realizar o diagnóstico e a quantificação da disfunção ventricular de maneira rápida e nãoinvasiva (Atkins e Snyder, 1992; Tei et al., 1997; Cavalcanti et al., 2006; Carvalho et al., 2007). 
Diversos índices ecoDopplercardiográficos têm sido utilizados para a avaliação da função ventricular. Entre as principais limitações ao emprego desses índices está a variação entre raças de diferentes portes, o que implica a necessidade de valores próprios para cada grupo racial (Atkins e Snyder, 1992; Morrison et al., 1992; Knigth, 1995; Snyder et al., 1995; Cavalcanti et al., 2007). Alguns índices são ainda influenciados pela freqüência cardíaca, pré-carga e pós-carga, podendo contribuir para erros de interpretação em alguns casos (Atkins e Snyder, 1992; Boon, 1998).

Recentemente foi proposto para o homem um índice que incorpora em uma só medida a performance sistólica e diastólica do ventrículo, denominado de índice de performance do miocárdio (IPM) (Tei et al., 1997). Na medicina humana, esse índice apresenta várias aplicações, tendo sido utilizado para avaliação prognóstica de pacientes com disfunção ventricular, na avaliação do teste de estresse do miocárdio e na estimativa da fração de ejeção do ventrículo esquerdo, entre outras (Lax et al., 2000; Harjai et al., 2002; Ling et al., 2003).

No levantamento bibliográfico realizado, foi encontrado apenas um trabalho que avaliou o uso do IPM em medicina veterinária, ao mostrar a sua utilidade no diagnóstico da miocardiopatia dilatada em cães da raça Terra Nova (Lee et al., 2002). Contudo, não foram realizados, até o momento, estudos que revelem se existem diferenças nos valores do IPM entre diferentes raças de cães, como demonstrado para diversos outros índices ecoDopplercardiográficos (Morrison et al., 1992; Snyder et al., 1995).

Esta pesquisa teve por objetivo avaliar a variação do IPM e de outros índices ecoDopplercardiográficos em duas raças caninas, Boxer e Schnauzer Miniatura, que apresentam alta prevalência de patologias cardíacas, mas que são de biótipos distintos.

\section{MATERIAL E MÉTODOS}

Foram utilizados 24 cães da raça Schnauzer Miniatura, 21 fêmeas e três machos, e 24 da raça Boxer, 19 fêmeas e cinco machos, com médias de pesos de $8,4 \pm 1,6 \mathrm{~kg}$ e $25,1 \pm 2,6 \mathrm{~kg}$, respectivamente. Os cães foram provenientes de diversos proprietários e criadores da região metropolitana de Belo Horizonte, MG, e foram selecionados dentro de uma faixa etária de dois a cinco anos de idade.

Todos os animais foram submetidos a exame clínico, eletrocardiográfico ${ }^{1}$ e radiográfico do tórax nas posições laterolateral e dorsoventral com o objetivo de excluir animais que apresentassem alterações cardiovasculares.

Os cães foram submetidos à sedação leve com maleato de acepromazina ${ }^{2}(0,03 \mathrm{mg} / \mathrm{kg})$ por via intravenosa, aguardando um período de descanso de 10 a 15 minutos. Após esse período, foram examinados em decúbito lateral em mesa apropriada, quando se fez necessária apenas uma leve contenção manual. O exame ecoDopplercardiográfico foi realizado utilizando-se um aparelho de ultra-sonografia ${ }^{3}$ composto pelos modos bidimensional, $\mathrm{M}$ e Doppler pulsado, contínuo e mapeamento em cores, com transdutor de varredura setorial mecânica de 2,5 a 7,5 MHz. Foram realizadas três a cinco medidas de cada índice em diferentes ciclos cardíacos.

Foram calculados os seguintes índices de função ventricular esquerda: IPM, percentual de encurtamento sistólico do ventrículo esquerdo $(\% \Delta D)$, fração de ejeção (Fej), velocidade circunferencial de encurtamento (Vcf), índice de volume sistólico final (IVsf), separação septal do ponto E (EPSS), tempo de ejeção (TE), índice de tempo de ejeção (ITE), período de pré-ejeção (PPE), relação entre período de pré-ejeção e o tempo de ejeção (PPE/TE), tempo de relaxamento isovolumétrico (TRIV), relação entre as ondas $\mathrm{E}$ e $\mathrm{A}$ do influxo mitral (E/A) e tempo de desaceleração da onda $\mathrm{E}$ do influxo mitral (Decel E).

Todos os posicionamentos e mensurações dos parâmetros ecoDopplercardiográficos, bem como cálculos dos diversos índices utilizados, seguiram as recomendações de Boon (1998). O cálculo do IPM foi realizado conforme proposto por Tei et al. (1997).

Utilizou-se análise de variância $(\mathrm{P}<0,05)$ e estimou-se a correlação entre índices

\footnotetext{
${ }^{1}$ Eletrocardiógrafo Ecafix ECG40 - Funbec

${ }^{2}$ Acepran $1 \%$ - Univet SA

${ }^{3}$ Ecocardiógrafo HP Sonos $100 \mathrm{CF}$ - Hewelwtt Packard
} 
ecoDopplercardiográficos e o peso corporal e a freqüência cardíaca (FC).

\section{RESULTADOS}

As freqüências cardíacas nos cães das raças Schnauzer Miniatura e Boxer, no momento do exame ecoDopplercardiográfico, foram $103 \mathrm{e}$ 112 batimentos por minuto (bpm), e não foi observada diferença significativa entre as raças.
Na Tab. 1, mostram-se as médias de cada um dos índices avaliados. Houve diferenças entre raças e observou-se correlação entre o peso corporal e esses mesmos índices (Tab. 2). Apenas foi observada correlação significativa entre a freqüência cardíaca e o TRIV $(\mathrm{r}=-0,38)$, TE $(\mathrm{r}=$ $-0,51)$ e PPE $(r=-0,44)$.

Tabela 1. Índices ecoDopplercardiográficos de função ventricular esquerda, avaliados em 48 cães das raças Schnauzer Miniatura e Boxer

\begin{tabular}{lcc}
\multicolumn{1}{c}{ Índices } & $\begin{array}{c}\text { Schnauzer Miniatura } \\
(\mathrm{n}=24)\end{array}$ & $\begin{array}{c}\text { Boxer } \\
(\mathrm{n}=24)\end{array}$ \\
\hline Índice de performance do miocárdio & $0,32 \pm 0,11 \mathrm{a}$ & $0,48 \pm 0,14 \mathrm{~b}$ \\
Índices da fase de ejeção & & \\
$\quad$ encurtamento sistólico do ventrículo esquerdo (\%) & $42,11 \pm 4,3 \mathrm{a}$ & $35,35 \pm 3,2 \mathrm{~b}$ \\
$\quad$ fração de ejeção & $75,08 \pm 5 \mathrm{a}$ & $65,28 \pm 4,1 \mathrm{~b}$ \\
$\quad$ velocidade circunferencial de encurtamento (cic/sec) & $2,35 \pm 0,35 \mathrm{a}$ & $2 \pm 0,28 \mathrm{~b}$ \\
$\quad$ volume sistólico final (ml/m²) & $14 \pm 6,7 \mathrm{a}$ & $27 \pm 8,6 \mathrm{~b}$ \\
separação septal do ponto E (mm) & $3,1 \pm 0,9 \mathrm{a}$ & $5,0 \pm 1,4 \mathrm{~b}$ \\
Intervalos de tempo sistólicos & $181 \pm 13 \mathrm{a}$ & $179 \pm 15 \mathrm{a}$ \\
tempo de ejeção (ms) & $233,9 \pm 10,1 \mathrm{a}$ & $231,5 \pm 14,4 \mathrm{a}$ \\
$\quad$ índice de tempo de ejeção (ms) & $57 \pm 7 \mathrm{a}$ & $75 \pm 10 \mathrm{~b}$ \\
$\quad$ período de pré-ejeção (ms) & $0,32 \pm 0,05 \mathrm{a}$ & $0,42 \pm 0,06 \mathrm{~b}$ \\
período de pré-ejeção/tempo de ejeção & & \\
Índices de função diastólica & $67 \pm 5 \mathrm{a}$ & $73 \pm 10 \mathrm{a}$ \\
tempo de relaxamento isovolumétrico (ms) & $1,6 \pm 0,3 \mathrm{a}$ & $1,6 \pm 0,3 \mathrm{a}$ \\
relação entre as ondas E e A do influxo mitral & $77 \pm 10 \mathrm{a}$ & $98 \pm 18 \mathrm{~b}$ \\
tempo de desaceleração da onda E do influxo mitral (ms) & &
\end{tabular}

Letras diferentes na mesma linha indicam diferença entre valores $(\mathrm{P}<0,01)$.

Tabela 2. Correlações entre o peso corporal e os índices EcoDopplercardiográficos de função ventricular esquerda, em cães das raças Boxer e Schnauzer Miniatura

\begin{tabular}{lc}
\multicolumn{1}{c}{ Índices } & Correlação $\left(\mathrm{r}^{*}\right)$ \\
\hline Índice de performance do miocárdio & 0,53 \\
Índices da fase de ejeção & $-0,63$ \\
encurtamento sistólico do ventrículo esquerdo (\%) & $-0,70$ \\
fração de ejeção & $-0,45$ \\
velocidade circunferencial de encurtamento (cic/sec) & 0,60 \\
volume sistólico final (ml/ $\left.\mathrm{m}^{2}\right)$ & 0,65 \\
separação septal do ponto E (mm) & $\mathrm{ns}$ \\
Intervalos de tempo sistólicos & $\mathrm{ns}$ \\
tempo de ejeção (ms) & 0,62 \\
índice de tempo de ejeção (ms) & 0,65 \\
período de pré-ejeção (ms) & \\
período de pré-ejeção/tempo de ejeção & $\mathrm{ns}$ \\
Índices de função diastólica & $\mathrm{ns}$ \\
tempo de relaxamento isovolumétrico (ms) & 0,63 \\
relação entre as ondas E e A do influxo mitral & \\
tempo de desaceleração da onda E do influxo mitral (ms) & \\
\hline
\end{tabular}




\section{DISCUSSÃO}

O peso corporal parece ser a principal característica racial responsável pelas diferenças observadas entre as raças Schnauzer Miniatura e Boxer. $\mathrm{O}$ uso de índices de referência apropriados a cada faixa de peso é importante no exame ecoDopplercardiográfico. Contudo, o peso corporal não deve ser utilizado como a única explicação. De acordo com Morrison et al. (1992) e Snyder et al. (1995), outros fatores como a geometria ventricular, a relação entre o peso do coração e o peso corporal, e a conformação torácica também devem ser levados em consideração.

Os valores do IPM encontrados na raças Schnauzer Miniatura e Boxer são os primeiros descritos para essas raças na literatura veterinária. Como só foram utilizados animais sadios, são necessários estudos com animais portadores de patologias cardíacas para se estabelecer limites entre cães sadios e doentes. Este estudo indica a necessidade de se estabelecer melhor os valores normais do IPM para os diferentes grupos raciais antes de utilizálo na rotina clínica.

A baixa correlação observada entre o IPM e a FC já havia sido descrita em estudos anteriores (Poulsen et al., 2000; Lee et al., 2002). Essa independência em relação à $\mathrm{FC}$ deve-se ao fato do IPM ser derivado do tempo de contração isovolumétrica e do tempo de ejeção, duas medidas que são inversamente proporcionais à FC. Como um se encontra no numerador e outro no denominador da fórmula do IPM, há uma regulação automática em relação às mudanças na FC.

A não correlação entre os índices de fase de ejeção e a FC era esperada, pois esses índices são baseados em medidas anatômicas, que aparentemente são pouco influenciados pelo estado cronotrópico. Deve-se ressaltar que as freqüências cardíacas observadas mantiveram-se nos em limites fisiológicos. Segundo Jacobs e Mahjoob (1988), as alterações de FC decorrentes de processos patológicos ou farmacológicos podem alterar os índices funcionais, pois estão associadas a uma complexa interação de mecanismos cardiovasculares.
Dos quatro intervalos temporais avaliados, TE, PPE, TRIV e Decel E, três mostraram correlação com a $\mathrm{FC}$, o que reforça a idéia que esses intervalos, mais que as medidas anatômicas, são influenciados pela $\mathrm{FC}$, o que é esperado, uma vez que tanto o tempo de sístole como o de diástole variam inversamente proporcional à $\mathrm{FC}$.

Pode-se utilizar o ITE para corrigir a variação imposta pela freqüência cardíaca sobre o TE. O uso desse artifício mostrou-se eficaz, uma vez que neste trabalho as ITE derivadas dos tempos de ejeção não apresentaram correlação significativa com a FC.

A relação $\mathrm{PPE} / \mathrm{TE}$ não foi influenciada pela $\mathrm{FC}$ como o foram o PPE ou o TE, tornando-a uma boa alternativa na comparação entre animais com diferentes FC ou em exames seriados de um mesmo paciente. Segundo Spoidick et al. (1984), a relação PPE/TE é maior com outros índices de função do que o TE ou PPE em separado, o que reforça as vantagens de sua utilização.

A relação E/A do influxo mitral foi a característica de menor variabilidade entre os índices de função diastólica, não apresentando diferença entre as raças ou correlação com o peso corporal ou FC. Essas características são de grande importância, pois possibilitam o uso de seus valores de referência em animais de diferentes raças e em diferentes estados conotrópicos.

\section{REFERÊNCIAS BIBLIOGRÁFICAS}

ATKINS, C.E.; SNYDER, P.S. Systolic time intervals and their derivatives for evaluation of cardiac function. J. Vet. Intern. Med., v.6, p.5563, 1992.

BOON, J. A. Evaluation of size, function, and hemodynamics. In: Manual of veterinary echocardiography. Baltimore: Willians e Wilkins, 1998. cap.3, p.151-260.

CARVALHO, R.O.; ARAÚJO, R.B.; SILVA, E.F. Ecocardiografia modo Doppler pulsado em gatos clinicamente sadios. Arq. Bras. Med. Vet. Zootec., v.58, p.333-340, 2006.

CAVALCANTI, G.A.O; MUZZI, R.A.L.; ARAÚJO, R.B. et al. Avaliação ecodopplercardiográfica da função diastólica em 
cães da raça Boxer. Arq. Bras. Med. Vet. Zootec., v.59, p.1169-1176, 2007.

HARJAI, K.J.; SCOTT, L.; VIVEKANANTHAN, K. et al. The Tei index: a new prognostic index for patients with symptomatic heart failure. J. Am. Soc. Echoc., v.15, p.864-868, 2002.

JACOBS, G.; MAHJOOB, K. Influence of alterations in heart rate on echocardiographic measurements in the dog. Am. J. Vet. Res., v.49, p.548- 552, 1988.

KNIGHT, D.H. Fisiopatologia da insuficiência cardíaca e avaliação clínica da função cardíaca In: ETTINGER, S.J.; FELDMAN, E.C. (Eds). Tratado de medicina interna Veterinária. 4.ed. São Paulo: Manole, 1995. v.1, cap.91, p.11851217.

LAX, J.A.; BERMANN, A.M.; CIANCIULLI, T.F. et al. Estimation of the ejection fraction in patients with myocardial infarction obtained from the combined index of systolic and diastolic left ventricular function: a new method. J. Am. Soc. Echoc., v.13, p.116-123, 2000.

LEE, B.H.; McEWAN, J. D.; FRENCH, A. T. et al. Evaluation of a novel doppler index of combined systolic and diastolic myocardial performance in newfoudland dogs with familial prevalence of dilated cardiomyopathy. Vet. Rad. Ultras., v.43, p.154-165, 2002.

LING, L.H.; TEI, C.; MCCULLY, R.B. et al. Analysis of systolic and diastolic time intervals during dobutamine-atropine stress echocardiography: diagnostic potential of the Doppler myocardial performance index. J. Am. Soc. Echoc., v.14, p.978-986, 2003.

MORRISON, S.A.; MOISE, N.S.; SCARLETT, J. et al. Effect of breed and body weight on echocardiographic values in four breeds of dogs of differing somatotype. J. Vet. Intern. Med., v.6, p.220-224, 1992.

POULSEN, S.H.; NIELSEN, J.C.; ANDERSEN, H.R. The influence of heart rate on the Dopplerderived myocardial performance index. J. Am. Soc. Echoc., v.13, p.379-384, 2000.

SNYDER, P.S.; SATO, T.; ATKINS, C.E. A comparison of echocardiographic indices of the nonracing, healthy greyhound to reference values from other breeds. Vet. Rad. Ultrasound, v.36, p.387-392, 1995.

SPOIDICK, D.H.; DOI, Y.L.; BISHOP, R.L. et al. Systolic time intervals reconsidered. Reevaluation of the preejection period: absence of relation to heart rate. Am. J. Cardiol., v.53, p.1667-1670, 1984.

TEI, C.; NISHIMURA, R.A.; SEWARD, J.B. et al. Noninvasive Doppler-derived myocardial performance index: correlation with simultaneous measurements of cardiac catheterization measurements. J. Am. Soc. Echoc., v.10, p.169-178, 1997. 